\section{The Health Consequences of Poverty in Canada}

\author{
Jeff Turnbull, MD, FRCP(C) \\ Tiina Podymow, MD, CM, FRCP(C)
}

$\mathrm{P}$ overty remains prevalent in Canada despite social and economic prosperity. Vulnerable populations including new immigrants, Aboriginal peoples, single parent families and the elderly constitute a growing percentage of the lower extreme of the socioeconomic scale. Homelessness has been declared a national crisis with conservative estimates exceeding 5 per 10,000, numbers unheard of in previous years. ${ }^{1}$ Emergency shelters and food programs are no longer visited solely by adult males with psychiatric disease or addictions, as single parent families and the elderly represent a growing segment seeking help. In almost every major urban centre in Canada, there are children who have known no other housing than that of an emergency shelter.

Socioeconomic status as an important determinant of health is well documented. ${ }^{2}$ The poor and particularly the homeless suffer from increased morbidity and mortality, with age- and sex-adjusted rates varying between 2.3 to 31 times that of the national average. ${ }^{3,4}$ What has not been well defined are the true consequences and social costs of poverty. Despite national attention placed upon poverty and the homeless, human and fiscal costs while substantial - are yet unknown.

While drug addiction, alcoholism and psychiatric disease are prevalent, illnesses common in the general population such as vascular disease, cancer and degenerative diseases are also present with increased frequency in the homeless population. Trauma, physical and sexual assault, and infections such as HIV/AIDS, viral hepatitis and tuberculosis are especially common. ${ }^{5}$ In addition to having a greater burden of illness, the homeless do not have the same access to health services as other Canadians. Factors such as geographic isolation, lack of transportation, stigmatization, lack of information and mistrust are barriers; chronic diseases often go untreated or unrecognized. When the poor do access health services, it is frequently for crisis management and has ensuing difficulties with treatment, compliance and follow-up. ${ }^{1}$

While the extent and nature of poverty in Canada have been described, further complications experienced by the poor are characterized in articles by Hwang: Is Homelessness Hazardous to Your Health? ${ }^{6}$ and McIntyre et al.: Food Insecurity of Low-income Lone Mothers and Their Children in Atlantic Canada. ${ }^{7}$

Hwang investigates the association between homelessness and mortality. Compared with the general population, the homeless suffer from a variety of medical and psychiatric illnesses leading to dramatically increased morbidity and mortality, and in this longitudinal study increased mortality was related to episodes of shelter use. However, as Hwang noted, a direct causal relationship between episodes of homelessness and mortality is yet to be proven and ultimately, may never be found. This implies that the solution to homelessness is not as easy as simply providing housing or treating the coexisting medical or psychiatric illnesses in isolation. The best management may be in overcoming the barriers to providing health services, and by making these services available within the shelters themselves. Currently under study, this approach has the advantage of ongoing rapport, integration of primary care into existing shelter services, follow-up, and connections to tertiary care. ${ }^{8-10}$

Insufficient nutrition is also a consequence of poverty. ${ }^{11,12}$ In the article by McIntyre et al., low income, mother-led households in Atlantic Canada experienced difficulty obtaining food in almost all households (96.5\%). These findings have been supported in previous Canadian studies. ${ }^{13,14}$ Child and maternal hunger occurred at a rate above that of the national average (23\%) and elderly mothers were at higher risk, which is different from some national

Faculty of Medicine, University of Ottawa

Correspondence: Dr. Jeff Turnbull, Chairman, Department of Medicine, The Ottawa Hospital General Campus, 501 Smyth Road, Room LM12, Ottawa, ON K1H 8L6

NOVEMBER - DECEMBER 2002 studies. ${ }^{14}$ In Nova Scotia and Prince Edward Island, households were noted to be at increased risk, which has implications for those who set social policy. Additional important questions remain concerning the nutritional quality of the cohort's diet, priorities in the household budgets, and health indicators such as anemia.

These two articles highlight important methodologic issues concerning poverty-related research. Study cohorts are difficult to describe (sheltered vs. unsheltered vs. unstably housed), difficult to sample, heterogeneous and have multiple confounding influences: they can be transient, non-compliant, have many co-morbidities and be unreliable historians. Social services and cultural differences differ among provinces and regions, making the generalizability of results difficult. Additionally, this is interdisciplinary research and includes qualitative and quantitative methodologies. Despite the challenges of this research, there is a pressing need for applied clinical research to guide public policy and to direct patient care. Meanwhile, health practitioners can better care for their own patients by recognizing the health risks that exist in the most vulnerable segments of society.

\section{REFERENCES/REFÉRENCES}

1. Hwang SW. Homelessness and health. CMAJ 2001;164(2):229-33.

2. Marmot M. Introduction. In: Marmot M, Wilkinson RG (Eds.) Social Determinants of Health. Great Britain: Oxford University Press, 2001;1-16.

3. Hwang SW. Mortality among men using homeless shelters in Toronto, Ontario. JAMA 2000;283(16):2152-57.

4. Roy E, Boivin F, Haley N, Lemire N. Mortality among street youth. Lancet 1998;352(9121):32.

5. Gelberg L, Linn LS. Assessing the physical health of homeless adults. JAMA 1989;262(14):1973-79.

6. Hwang SW. Is homelessness hazardous to your health? Obstacles to the demonstration of a causal relationship. CJPH 2002;93(6):407-10.

7. McIntyre L, Glanville NT, Officer S, Anderson B, Raine KD, Dayle JB. Food insecurity of lowincome lone mothers and their children in Atlantic Canada. CJPH 2002;93(6):411-15.

8. Inner City Health Project 2001. www.med. uottawa.ca/ichpsuo/

9. Redlener I. Healthcare for the homeless-lessons from the front line. NEJM 1994(5);331:327-28.

10. Lechky O. Bringing healthcare to the homeless. CMAJ 1999;161(1):13.

11. Robertson A, Brunner E, Sheiham A. Food is a political issue. In: Marmot M, Wilkinson RG (Eds.) Social Determinants of Health. Great Britain: Oxford University Press, 2001;179-210.

12. Wiecha JL, Dwyer JT, Dunn-Strohecker M. Nutrition and health services needs among the homeless. Public Health Rep 1991(106);4:364-74.

13. Canada's Action Plan for Food Security, Agriculture and Agri-Food Canada. http://www.agr.gc.ca/ $\mathrm{misb} /$ fsb/fsap/part2e.html\#2.2

14. Food Insecurity in Canadian Households. Statistics Canada 2001. http://www.statcan.ca/ Daily/English/010815/d010815a.htm 


\section{Les conséquences sanitaires de la pauvreté au Canada}

\author{
Jeff Turnbull, MD, FRCP \\ Tiina Podymow, MD, CM, FRCP
}

$\mathrm{L}$

a pauvreté règne encore au Canada malgré la prospérité sociale et économique. Les populations vulnérables - nouveaux immigrants, Autochtones, familles monoparentales et personnes âgées - sont de plus en plus présentes au bas de l'échelle. Le nombre de sans-abri atteint des proportions critiques partout au pays; selon les estimations prudentes, ils seraient plus de 5 pour 10000 , un seuil sans précédent. ${ }^{1}$ Les maisons de refuge et les banques d'alimentation ne sont plus seulement fréquentées par des hommes d'âge adulte atteints de maladies psychiatriques ou de toxicomanies : les familles monoparentales et les personnes âgées sont proportionnellement plus nombreuses qu'auparavant à recourir à cette forme d'aide. Dans presque tous les grands centres urbains du Canada, il $\mathrm{y}$ a des enfants qui n'ont jamais eu d'autre foyer qu'une maison de refuge.

Le fait que le statut socio-économique soit un déterminant important de la santé est bien documenté. ${ }^{2}$ Les pauvres, tout particulièrement les sans-abri, ont des taux de morbidité et de mortalité accrus, entre 2,3 et 31 fois la moyenne nationale lorsqu'on tient compte de l'âge et du sexe. ${ }^{3,4}$ Mais on connaît beaucoup moins bien les conséquences et les coûts sociaux véritables de la pauvreté. Bien que l'on s'intéresse à la pauvreté et à la clochardise au palier fédéral, on en ignore encore les coûts humains et financiers - qui sont considérables.

On connaît la prédominance de la toxicomanie, de l'alcoolisme et des maladies psychiatriques chez les sans-abri, mais les maladies courantes dans la population générale (affections vasculaires, cancers, maladies dégénératives) sont aussi de plus en plus fréquentes chez eux. Les traumatismes, les agressions physiques et sexuelles et les infections (VIH/sida, hépatite virale, tuberculose) sont particulièrement répandus. ${ }^{5}$ Plus souvent malades, les sans-abri ont moins accès aux services de santé que les autres Canadiens. L'isolement géographique, le manque de moyens de transport, la stigmatisation, le manque d'information et la méfiance y font obstacle; souvent, donc, leurs maladies chroniques ne sont ni diagnostiquées, ni traitées. Lorsque les pauvres obtiennent des services de santé, c'est souvent en situation de crise, ce qui pose ensuite des problèmes de traitement, d'assiduité et de suivi. ${ }^{1}$

L'envergure et la nature de la pauvreté au Canada ont été décrites, mais on manquait jusqu'ici d'informations sur les complications supplémentaires vécues par les pauvres, décrites dans les articles de Hwang, "Is Homelessness Hazardous to Your Health? " et de McIntyre et al., "Food Insecurity of Low-income Lone Mothers and Their Children in Atlantic Canada. $»^{7}$

Hwang a étudié les liens entre la clochardise et la mortalité. Par rapport à la population générale, les sans-abri souffrent de diverses maladies physiques et psychiatriques qui font grimper de façon spectaculaire leurs taux de morbidité et de mortalité; dans cette étude longitudinale, les épisodes d'hébergement en maison de refuge sont liés à une mortalité accrue. Cependant, comme le remarque Hwang, l'existence d'une relation causale directe entre les épisodes de clochardise et la mortalité n'est pas encore prouvée et pourrait ne jamais l'être. La solution au phénomène des sans-abri pourrait donc ne pas être aussi simple que d'offrir des logements ou de traiter isolément des maladies physiques ou psychiatriques coexistantes. Le mieux serait peut-être de surmonter les obstacles à l'accès aux services de santé en offrant ces services à l'intérieur même des maisons de refuge. Une telle approche, actuellement à l'étude, favoriserait la continuité des rapports, l'intégration des soins primaires dans les services existants des maisons de refuge, le suivi, ainsi que l'aiguillage vers les soins tertiaires. ${ }^{8-10}$

Les carences nutritionnelles sont une autre conséquence de la pauvreté. ${ }^{11,12}$ Dans l'article de McIntyre et al., les ménages à faibles revenus du Canada atlantique dirigés par des mères éprouvaient presque tous $(96,5 \%)$ des difficultés à se procurer de la nourriture. Des études canadiennes précédentes confirment ces résultats. ${ }^{13,14}$ À la différence de certaines études nationales cependant, on a constaté que les enfants et les mères avaient faim dans une proportion supérieure à la moyenne nationale (23\%), et que les mères âgées étaient plus vulnérables. ${ }^{14}$ Les ménages les plus à risque étaient ceux de la Nouvelle-Écosse et de l'Île-du-Prince-Édouard, un résultat qui a des conséquences pour les décideurs des politiques sociales. Il subsiste d'autres questions importantes concernant la qualité nutritive de la diète de la cohorte, les priorités budgétaires des ménages et les indicateurs de santé comme l'anémie.

Ces deux articles mettent en lumière d'importants problèmes méthodologiques des études sur la pauvreté. Les cohortes étudiées sont difficiles à décrire (les sujets vivent-ils dans un logement stable? précaire? inexistant?), difficiles à échantillonner et hétérogènes. De multiples facteurs confusionnels entrent en jeu, car les sujets peuvent être itinérants, indociles, souffrir de nombreux troubles différents ou avoir mauvaise mémoire. Les services sociaux et les pratiques culturelles differrent d'une province et d'une région à l'autre, ce qui complique la généralisation des résultats. Enfin, comme dans toute recherche interdisciplinaire, il faut combiner des méthodes qualitatives et quantitatives. Malgré ces défis, il existe un besoin pressant d'études cliniques appliquées pour orienter les politiques gouvernementales et les soins directs aux malades. Entre-temps, les professionnels de la santé qui veulent mieux prendre soin de leurs propres patients doivent reconnaitre les risques sanitaires auxquels sont exposés les membres les plus vulnérables de la société. 